\title{
Bispectrality of the Complementary Bannai-Ito Polynomials
}

\author{
Vincent X. GENEST ${ }^{\dagger}$, Luc VINET ${ }^{\dagger}$ and Alexei ZHEDANOV ${ }^{\ddagger}$ \\ † Centre de Recherches Mathématiques, Université de Montréal, \\ C.P. 6128, Succursale Centre-ville, Montréal, Québec, Canada, H3C 3J7 \\ E-mail: genestvi@crm.umontreal.ca,luc.vinet@umontreal.ca \\ $\ddagger$ Donetsk Institute for Physics and Technology, Ukraine \\ E-mail: zhedanov@yahoo.com
}

Received November 13, 2012, in final form February 27, 2013; Published online March 02, 2013 http://dx.doi.org/10.3842/SIGMA.2013.018

\begin{abstract}
A one-parameter family of operators that have the complementary BannaiIto (CBI) polynomials as eigenfunctions is obtained. The CBI polynomials are the kernel partners of the Bannai-Ito polynomials and also correspond to a $q \rightarrow-1$ limit of the AskeyWilson polynomials. The eigenvalue equations for the CBI polynomials are found to involve second order Dunkl shift operators with reflections and exhibit quadratic spectra. The algebra associated to the CBI polynomials is given and seen to be a deformation of the Askey-Wilson algebra with an involution. The relation between the CBI polynomials and the recently discovered dual -1 Hahn and para-Krawtchouk polynomials, as well as their relation with the symmetric Hahn polynomials, is also discussed.
\end{abstract}

Key words: Bannai-Ito polynomials; quadratic algebras; Dunkl operators

2010 Mathematics Subject Classification: 33C02; 16G02

\section{Introduction}

One of the recent advances in the theory of orthogonal polynomials (OPs) has been the discovery of several new families of "classical" OPs that correspond to $q \rightarrow-1$ limits of $q$-polynomials of the Askey scheme [20, 21, 25, 26]. The word "classical" here refers to the fact that in addition to obeying the three-term relation

$$
\mathcal{P}_{n+1}(x)+\beta_{n} \mathcal{P}_{n}(x)+\gamma_{n} \mathcal{P}_{n-1}(x)=x \mathcal{P}_{n}(x),
$$

the polynomials $\mathcal{P}_{n}(x)$ also satisfy an eigenvalue equation of the form

$$
\mathcal{L} \mathcal{P}_{n}(x)=\lambda_{n} \mathcal{P}_{n}(x)
$$

The novelty of these families of -1 orthogonal polynomials lies in the fact that for each family the operator $\mathcal{L}$ is a differential or difference operator that also contains the reflection operator $R f(x)=f(-x)$ [24]. Such differential/difference operators are said to be of Dunkl type [4], notwithstanding the fact that the operators $\mathcal{L}$ differ from the standard Dunkl operators in that they preserve the linear space of polynomials of any given maximal degree. In this connection, these -1 OPs have also been referred to as Dunkl orthogonal polynomials.

With the discovery and characterization of these Dunkl polynomials, a -1 scheme of OPs, completing the Askey scheme, is beginning to emerge. At the top of the discrete variable branch of this -1 scheme lie two families of orthogonal polynomials: the Bannai-Ito (BI) polynomials and their kernel partners the complementary Bannai-Ito polynomials (CBI); both families correspond to different $q \rightarrow-1$ limits of the Askey-Wilson polynomials. 
The Bannai-Ito polynomials were originally identified by Bannai and Ito themselves in [1] where they recognized that these OPs correspond to the $q \rightarrow-1$ limit of the $q$-Racah polynomials. However, it is only recently [21] that the Dunkl shift operator $\mathcal{L}$ admitting the BI polynomials as eigenfunctions has been constructed. The BI polynomials and their special cases enjoy the Leonard duality property, a property they share with all members of the discrete part of the Askey scheme $[1,14]$. This means that in addition to satisfying a three-term recurrence relation, the BI polynomials also obey a three-term difference equation. From the algebraic point of view, this property corresponds to the existence of an associated Leonard pair [18].

Amongst the discrete-variable -1 polynomials, there are families that do not possess the Leonard duality property. That is the case of the complementary Bannai-Ito polynomials and their descendants [20,21]. This situation is connected to the fact that in these cases the difference operator of the corresponding $q$-polynomials do not admit a $q \rightarrow-1$ limit. In [20], a five-term difference equation was nevertheless constructed for the dual -1 Hahn polynomials and the defining Dunkl operator for these polynomials was found.

In this paper, a one-parameter family of Dunkl operators $\mathcal{D}_{\alpha}$ of which the complementary Bannai-Ito polynomials are eigenfunctions is derived, thus establishing the bispectrality of the CBI polynomials. The operators of this family involve reflections and are of second order in discrete shifts; they are diagonalized by the CBI polynomials with a quadratic spectrum. The corresponding five-term difference equation satisfied by the CBI polynomials is presented. Moreover, an algebra associated to the CBI polynomials is derived. This quadratic algebra, called the complementary Bannai-Ito algebra, is defined in terms of four generators. It can be seen as a deformation with an involution of the quadratic Hahn algebra QH(3) [8,31], which is a special case of the Askey-Wilson AW(3) algebra [17, 29].

The paper, which provides a comprehensive description of the CBI polynomials and their properties, is organized in the following way. In Section 2, we present a review of the BannaiIto polynomials. In Section 3, we define the complementary Bannai-Ito polynomials and obtain their recurrence and orthogonality relations. In Section 4, we use a proper $q \rightarrow-1$ limit of the Askey-Wilson difference operator to construct an operator $\mathcal{D}$ of which the CBI polynomials are eigenfunctions. We use a "hidden" eigenvalue equation to show that one has in fact a oneparameter family of operators $\mathcal{D}_{\alpha}$, parametrized by a complex number $\alpha$, that is diagonalized by the CBI polynomials. In Section 5, we derive the CBI algebra and present some aspects of its irreducible representations. In Section 6, we discuss the relation between the CBI polynomials and three other families of OPs: the dual -1 Hahn, the para-Krawtchouk and the classical Hahn polynomials; these OP families are respectively a limit and two special cases of the CBI polynomials. We conclude with a perspective on the continuum limit and an outlook.

\section{Bannai-Ito polynomials}

The Bannai-Ito polynomials were introduced in 1984 [1] in the complete classification of orthogonal polynomials possessing the Leonard duality property (see Section 4). It was shown that they can be obtained as a $q \rightarrow-1$ limit of the $q$-Racah polynomials and some of their properties were derived. Recently [21], it was observed that the BI polynomials also occur as eigensolutions of a first order Dunkl shift operator. In the following, we review some of the properties of the BI polynomials; we use the presentation of [21].

The monic BI polynomials $B_{n}\left(x ; \rho_{1}, \rho_{2}, r_{1}, r_{2}\right)$, denoted $B_{n}(x)$ for notational convenience, satisfy the three-term recurrence relation

$$
B_{n+1}(x)+\left(\rho_{1}-A_{n}-C_{n}\right) B_{n}(x)+A_{n-1} C_{n} B_{n-1}(x)=x B_{n}(x),
$$

with the initial conditions $B_{-1}(x)=0$ and $B_{0}(x)=1$. The recurrence coefficients $A_{n}$ and $C_{n}$ 
are given by

$$
\begin{aligned}
& A_{n}= \begin{cases}\frac{\left(n+2 \rho_{1}-2 r_{1}+1\right)\left(n+2 \rho_{1}-2 r_{2}+1\right)}{4(n+g+1)}, & n \text { even, } \\
\frac{(n+2 g+1)\left(n+2 \rho_{1}+2 \rho_{2}+1\right)}{4(n+g+1)}, & n \text { odd },\end{cases} \\
& C_{n}= \begin{cases}-\frac{n\left(n-2 r_{1}-2 r_{2}\right)}{4(n+g)}, & n \text { even }, \\
-\frac{\left(n+2 \rho_{2}-2 r_{2}\right)\left(n+2 \rho_{2}-2 r_{1}\right)}{4(n+g)}, & n \text { odd },\end{cases}
\end{aligned}
$$

where

$$
g=\rho_{1}+\rho_{2}-r_{1}-r_{2} .
$$

It is seen from the above formulas that the positivity condition $u_{n}=A_{n-1} C_{n}>0$ cannot be satisfied for all $n \in \mathbb{N}$ [2]. Hence it follows that the Bannai-Ito polynomials can only form a finite set of positive-definite orthogonal polynomials $B_{0}(x), \ldots, B_{N}(x)$, which occurs when the "local" positivity condition $u_{i}>0$ for $i \in\{1, \ldots, N\}$ and the truncation conditions $u_{0}=0$, $u_{N+1}=0$ are satisfied. If these conditions are fulfilled, the BI polynomials $B_{n}(x)$ satisfy the discrete orthogonality relation

$$
\sum_{k=0}^{N} w_{k} B_{n}\left(x_{k}\right) B_{m}\left(x_{k}\right)=h_{n} \delta_{n m},
$$

with respect to the positive weight $w_{k}$. The spectral points $x_{k}$ are the simple roots of the polynomial $B_{N+1}(x)$. The explicit formulae for the weight function $w_{k}$ and the grid points $x_{k}$ depend on the realization of the truncation condition $u_{N+1}=0$.

If $N$ is even, it follows from (2.2) that the condition $u_{N+1}=0$ is tantamount to one of the following requirements:

$$
\begin{array}{ll}
\text { 1) } r_{1}-\rho_{1}=\frac{N+1}{2}, & \text { 2) } r_{2}-\rho_{1}=\frac{N+1}{2}, \\
\text { 3) } r_{1}-\rho_{2}=\frac{N+1}{2}, & \text { 4) } r_{2}-\rho_{2}=\frac{N+1}{2} .
\end{array}
$$

For the cases 1) and 2), the grid points have the expression

$$
x_{k}=(-1)^{k}\left(k / 2+\rho_{1}+1 / 4\right)-1 / 4,
$$

and the weights take the form

$$
w_{k}=\frac{(-1)^{\nu}}{\ell !} \frac{\left(\rho_{1}-r_{1}+1 / 2\right)_{\ell+\nu}\left(\rho_{1}-r_{2}+1 / 2\right)_{\ell+\nu}\left(\rho_{1}+\rho_{2}+1\right)_{\ell}\left(2 \rho_{1}+1\right)_{\ell}}{\left(\rho_{1}+r_{1}+1 / 2\right)_{\ell+\nu}\left(\rho_{1}+r_{2}+1 / 2\right)_{\ell+\nu}\left(\rho_{1}-\rho_{2}+1\right)_{\ell}},
$$

where one has $k=2 \ell+\nu$ with $\nu \in\{0,1\}$ and where $(a)_{n}=a(a+1) \cdots(a+n-1)$ is the Pochhammer symbol. For the cases 3 ) and 4), the formulae (2.4) and (2.5) hold under the substitution $\rho_{1} \leftrightarrow \rho_{2}$.

If $N$ is odd, it follows from (2.2) that the condition $u_{N+1}=0$ is equivalent to one of the following restrictions:
i) $\rho_{1}+\rho_{2}=-\frac{N+1}{2}$,
ii) $r_{1}+r_{2}=\frac{N+1}{2}$,
iii) $\rho_{1}+\rho_{2}-r_{1}-r_{2}=-\frac{N+1}{2}$. 
The condition $\mathrm{iii}$ ) leads to a singularity in $u_{n}$ when $n=(N+1) / 2$ and hence only the conditions $i$ ) and $i i$ ) are admissible. For the case $i$ ), the formulae (2.4) and (2.5) hold under the substitution $\rho_{1} \leftrightarrow \rho_{2}$. For the case $\left.i i\right)$, the spectral points are given by

$$
x_{k}=(-1)^{k}\left(r_{1}-k / 2-1 / 4\right)-1 / 4,
$$

and the weight function is given by (2.5) with the substitutions $\left(\rho_{1}, \rho_{2}, r_{1}, r_{2}\right) \rightarrow-\left(r_{1}, r_{2}, \rho_{1}, \rho_{2}\right)$.

The Bannai-Ito polynomials can be obtained from a $q \rightarrow-1$ limit of the Askey-Wilson polynomials and also have the Bannai-Ito algebra as their characteristic algebra (see [7] and [21]).

\section{CBI polynomials}

In this section we define the complementary Bannai-Ito polynomials through a Christoffel transformation of the Bannai-Ito polynomials. We derive their recurrence relation, hypergeometric representation and orthogonality relations from their kernel properties.

The complementary Bannai-Ito polynomials $I_{n}\left(x ; \rho_{1}, \rho_{2}, r_{1}, r_{2}\right)$, denoted $I_{n}(x)$ for convenience, are defined from the BI polynomials $B_{n}(x)$ by the transformation [21]

$$
I_{n}(x)=\frac{B_{n+1}(x)-A_{n} B_{n}(x)}{x-\rho_{1}},
$$

where $A_{n}$ is as in (2.2). The transformation (3.1) is an example of a Christoffel transformation [16]. It is easily seen from the definition (3.1) that $I_{n}(x)$ is a monic polynomial of degree $n$ in $x$. The inverse relation for the CBI polynomials is given by a Geronimus [30] transformation and has the expression

$$
B_{n}(x)=I_{n}(x)-C_{n} I_{n-1}(x) .
$$

This formula can be verified by direct substitution of (3.1) in (3.2) which yields back the defining relation (2.1) of the BI polynomials. In the reverse, the substitution of (3.2) in (3.1) yields the three-term recurrence relation [11]

$$
I_{n+1}(x)+\left(\rho_{1}-A_{n}-C_{n+1}\right) I_{n}(x)+A_{n} C_{n} I_{n-1}(x)=x I_{n}(x),
$$

where $A_{n}$ and $C_{n}$ are given by (2.2). The recurrence relation (3.3) can be written explicitly as

$$
I_{n+1}(x)+(-1)^{n} \rho_{2} I_{n}(x)+\tau_{n} I_{n-1}(x)=x I_{n}(x),
$$

where $\tau_{n}$ is given by

$$
\begin{aligned}
& \tau_{2 n}=-\frac{n\left(n+\rho_{1}-r_{1}+1 / 2\right)\left(n+\rho_{1}-r_{2}+1 / 2\right)\left(n-r_{1}-r_{2}\right)}{(2 n+g)(2 n+g+1)}, \\
& \tau_{2 n+1}=-\frac{(n+g+1)\left(n+\rho_{1}+\rho_{2}+1\right)\left(n+\rho_{2}-r_{1}+1 / 2\right)\left(n+\rho_{2}-r_{2}+1 / 2\right)}{(2 n+g+1)(2 n+g+2)},
\end{aligned}
$$

and where $g=\rho_{1}+\rho_{2}-r_{1}-r_{2}$. One has also the initial conditions $I_{0}=1$ and $I_{1}=x-\rho_{2}$. The CBI polynomials are kernel polynomials of the BI polynomials. Indeed, by noting that

$$
A_{n}=B_{n+1}\left(\rho_{1}\right) / B_{n}\left(\rho_{1}\right),
$$

which follows by induction from (2.1), the transformation (3.1) may be cast in the form

$$
I_{n}(x)=\left(x-\rho_{1}\right)^{-1}\left[B_{n+1}(x)-\frac{B_{n+1}\left(\rho_{1}\right)}{B_{n}\left(\rho_{1}\right)} B_{n}(x)\right] .
$$


It is manifest from (3.6) that $I_{n}(x)$ are the kernel polynomials associated to $B_{n}(x)$ with kernel parameter $\rho_{1}$ [2]. Since the BI polynomials $B_{n}(x)$ are orthogonal with respect to a linear functional $\sigma^{(i)}$ :

$$
\left\langle\sigma^{(i)}, B_{n}(x) B_{m}(x)\right\rangle=0, \quad n \neq m
$$

where the upper index on $\sigma^{(i)}$ designates the possible functionals associated to the various truncation conditions, it follows from (3.6) that we have [2]

$$
\left\langle\sigma^{(i)},\left(x-\rho_{1}\right) I_{n}(x) I_{m}(x)\right\rangle=0, \quad n \neq m .
$$

Hence the orthogonality and positive-definiteness of the CBI polynomials can be studied using the formulae (3.5) and (3.7).

It is seen from (3.5) that the condition $\tau_{n}>0$ cannot be ensured for all $n$ and hence the complementary Bannai-Ito polynomials can only form a finite system of positive-definite orthogonal polynomials $I_{0}(x), \ldots, I_{N}(x)$, provided that the "local" positivity $\tau_{n}>0, n \in\{1, \ldots, N\}$, and truncation conditions $\tau_{0}=0$ and $\tau_{N+1}=0$ are satisfied.

When $N$ is even, the truncation conditions $\tau_{0}=0$ and $\tau_{N+1}=0$ are equivalent to one of the four prescriptions

$$
\begin{array}{ll}
\text { 1) } \rho_{2}-r_{1}=-\frac{N+1}{2}, & \text { 2) } \rho_{2}-r_{2}=-\frac{N+1}{2}, \\
\text { 3) } \rho_{1}+\rho_{2}=-\frac{N+2}{2}, & \text { 4) } g=-\frac{N+2}{2} .
\end{array}
$$

Since the condition 4) leads to a singularity in $\tau_{n}$, only the conditions 1), 2) and 3) are admissible. For all three conditions and assuming that the positivity conditions are satisfied, the CBI polynomials enjoy the orthogonality relation

$$
\sum_{k=0}^{N} \widetilde{w}_{k} I_{n}\left(x_{k}\right) I_{m}\left(x_{k}\right)=\widetilde{h}_{n} \delta_{n m},
$$

where the spectral points are given by

$$
x_{k}=(-1)^{k}\left(k / 2+\rho_{2}+1 / 4\right)-1 / 4,
$$

and the positive weights are

$$
\widetilde{w}_{k}=\left(x_{k}-\rho_{1}\right) w_{k},
$$

with $w_{k}$ defined by (2.5) with the substitution $\rho_{1} \leftrightarrow \rho_{2}$.

When $N$ is odd, the truncation conditions $\tau_{0}=0$ and $\tau_{N+1}=0$ are tantamount to

$$
\text { i) } \left.r_{1}-\rho_{1}=\frac{N+2}{2}, \quad \text { ii) } r_{1}+r_{2}=\frac{N+1}{2}, \quad \text { iii }\right) r_{2}-\rho_{1}=\frac{N+2}{2} \text {. }
$$

If the positivity condition $\tau_{n}>0$ is satisfied for $n \in\{1, \ldots, N\}$, the CBI polynomials will enjoy the orthogonality relation (3.9) with respect to the positive definite weight function $\widetilde{w}_{k}$. When either condition $i$ ) or $i i$ ) is satisfied, the spectral points are given by

$$
x_{k}=(-1)^{k}\left(r_{1}-k / 2-1 / 4\right)-1 / 4,
$$

together with the weight function $\widetilde{w}_{k}=\left(x_{k}-\rho_{1}\right) w_{k}$ where $w_{k}$ is given by $(2.5)$ with the replacement $\left(\rho_{1}, \rho_{2}, r_{1}, r_{2}\right)=-\left(r_{1}, r_{2}, \rho_{1}, \rho_{2}\right)$. Finally, the orthogonality relation for the truncation condition $\mathrm{iii}$ ) is obtained from the preceding case under the exchange $r_{1} \leftrightarrow r_{2}$. 
Let us now illustrate when positive-definiteness occurs for the CBI polynomials. We first consider the even $N$ case. It is sufficient to take

$$
\rho_{1}=\left(\frac{\frac{a+b}{2}+c+N}{2}\right), \quad \rho_{2}=\left(\frac{\frac{a+b}{2}-1}{2}\right), \quad r_{1}=\left(\frac{\frac{a+b}{2}+N}{2}\right), \quad r_{2}=\left(\frac{a-b}{4}\right),
$$

where $a, b$ and $c$ are arbitrary positive parameters. Assuming (3.11), the recurrence coefficients (3.5) become

$$
\tau_{n}= \begin{cases}\frac{n(N-n+a)(n+c+1)(n+b+c+N+1)}{16(n+g)(n+g+1)}, & n \text { even, } \\ \frac{(N-n+1)(n+b-1)(n+b+c)(n+a+b+c+N)}{16(n+g)(n+g+1)}, & n \text { odd }\end{cases}
$$

where $g=(b+c-1) / 2$. It is obvious from (3.12) that the positivity and truncation conditions are satisfied for $n \in\{1, \ldots, N\}$; this corresponds to the case 1) of (3.8).

Consider the situation when $N>1$ is odd. We introduce the parametrization

$$
\rho_{1}=\left(\frac{\frac{\zeta+\xi}{2}+\chi+N}{2}\right), \quad \rho_{2}=\left(\frac{\zeta-\xi}{4}\right), \quad r_{1}=\left(\frac{\frac{\zeta+\xi}{2}+N+1}{2}\right), \quad r_{2}=-\left(\frac{\zeta+\xi}{4}\right),
$$

where $\zeta, \xi$ and $\chi$ are arbitrary positive parameters. The recurrence coefficients become

$$
\tau_{n}= \begin{cases}\frac{n(N-n+1)(n+\chi)(n+\zeta+\xi+\chi+N+1)}{16(n+g)(n+g+1)}, & n \text { even, } \\ \frac{(N-n+\xi+1)(n+\zeta)(n+\zeta+\chi)(n+\zeta+\chi+N+1)}{16(n+g)(n+g+1)}, & n \text { odd }\end{cases}
$$

with $g=(\zeta+\chi-1) / 2$. Assuming (3.13), the positivity and truncation conditions are manifestly fulfilled; this corresponds to the condition $i$ ) of (3.10). The other cases can be treated in similar fashion.

It is possible to derive a hypergeometric representation for the CBI polynomials using a method [21, 25] which is analogous to Chihara's construction of symmetric orthogonal polynomials [2] and closely related to the scheme developed in [15] (see also [3]). Given the three-term recurrence relation (3.4), it follows by induction that the polynomials $I_{n}(x)$ can be written as

$$
I_{2 n}=R_{n}\left(x^{2}\right), \quad I_{2 n+1}=\left(x-\rho_{2}\right) Q_{n}\left(x^{2}\right),
$$

where $R_{n}\left(x^{2}\right)$ and $Q_{n}\left(x^{2}\right)$ are monic polynomials of degree $n$. It follows directly from (3.14) and (3.4) that the polynomials $R_{n}\left(x^{2}\right)$ and $Q_{n}\left(x^{2}\right)$ obey the following system of recurrence relations

$$
R_{n}(z)=Q_{n}(z)+\tau_{2 n} Q_{n-1}(z), \quad\left(z-\rho_{2}^{2}\right) Q_{n}(z)=R_{n+1}(z)+\tau_{2 n+1} R_{n}(z) .
$$

This system is equivalent to the following pair of equations:

$$
\begin{aligned}
& R_{n+1}(z)+\left(\rho_{2}^{2}+\tau_{2 n}+\tau_{2 n+1}\right) R_{n}(z)+\tau_{2 n-1} \tau_{2 n} R_{n-1}(z)=z R_{n}(z), \\
& Q_{n+1}(z)+\left(\rho_{2}^{2}+\tau_{2 n+1}+\tau_{2 n+2}\right) Q_{n}(z)+\tau_{2 n} \tau_{2 n+1} Q_{n-1}(z)=z Q_{n}(z) .
\end{aligned}
$$

These recurrence relations can be identified with those of the Wilson polynomials [13]. From this identification, we obtain

$$
R_{n}\left(x^{2}\right)=\eta_{n}{ }_{4} F_{3}\left[\begin{array}{c}
-n, n+g+1, \rho_{2}+x, \rho_{2}-x \\
\rho_{1}+\rho_{2}+1, \rho_{2}-r_{1}+1 / 2, \rho_{2}-r_{2}+1 / 2
\end{array} ; 1\right],
$$




$$
Q_{n}\left(x^{2}\right)=\iota_{n}{ }_{4} F_{3}\left[\begin{array}{c}
-n, n+g+2, \rho_{2}+1+x, \rho_{2}+1-x \\
\rho_{1}+\rho_{2}+2, \rho_{2}-r_{1}+3 / 2, \rho_{2}-r_{2}+3 / 2
\end{array} ; 1\right],
$$

where ${ }_{p} F_{q}$ denotes the generalized hypergeometric function [5] and where the normalization coefficients, which ensure that the polynomials are monic, are given by

$$
\begin{aligned}
& \eta_{n}=\frac{\left(\rho_{1}+\rho_{2}+1\right)_{n}\left(\rho_{2}-r_{1}+1 / 2\right)_{n}\left(\rho_{2}-r_{2}+1 / 2\right)_{n}}{(n+g+1)_{n}}, \\
& \iota_{n}=\frac{\left(\rho_{1}+\rho_{2}+2\right)_{n}\left(\rho_{2}-r_{1}+3 / 2\right)_{n}\left(\rho_{2}-r_{2}+3 / 2\right)_{n}}{(n+g+2)_{n}} .
\end{aligned}
$$

Thus the monic CBI polynomials have the hypergeometric representation (3.14). For definiteness and future reference, let us now gather the preceding results in the following proposition.

Proposition 1. The complementary Bannai-Ito polynomials $I_{n}\left(x ; \rho_{1}, \rho_{2}, r_{1}, r_{2}\right)$ are the kernel polynomials of the Bannai-Ito polynomials $B_{n}\left(x ; \rho_{1}, \rho_{2}, r_{1}, r_{2}\right)$ with kernel parameter $\rho_{1}$. The monic CBI polynomials obey the three-term recurrence relation

$$
I_{n+1}(x)+(-1)^{n} \rho_{2} I_{n}(x)+\tau_{n} I_{n-1}(x)=x I_{n}(x),
$$

where $\tau_{n}$ is given by (3.5). They have the explicit hypergeometric representation

$$
I_{2 n}(x)=R_{n}\left(x^{2}\right), \quad I_{2 n+1}(x)=\left(x-\rho_{2}\right) Q_{n}\left(x^{2}\right),
$$

where $R_{n}\left(x^{2}\right)$ and $Q_{n}\left(x^{2}\right)$ are as specified by (3.15). If the truncation condition $\tau_{N+1}=0$ and the positivity condition $\tau_{n}>0, n \in\{1, \ldots, N\}$, are satisfied, the CBI polynomials obey the orthogonality relation

$$
\sum_{k=0}^{N} \widetilde{w}_{k} I_{n}\left(x_{k}\right) I_{m}\left(x_{k}\right)=\widetilde{h}_{n} \delta_{n m},
$$

with respect to the positive weights $\widetilde{w}_{k}$. The grid points $x_{k}$ correspond to the simple roots of the polynomial $I_{N+1}(x)$. The formulas for the weights and grid points depend on the truncation condition. With $w_{k}\left(\rho_{1}, \rho_{2}, r_{1}, r_{2}\right)$ given as in $(2.5)$, one has

1. For $r_{1}=\frac{N+1}{2}+\rho_{2}, r_{2}=\frac{N+1}{2}+\rho_{2}$ or $\rho_{1}=-\frac{N+2}{2}-\rho_{2}$ with $N$ even:

$$
x_{k}=(-1)^{k}\left(\rho_{2}+k / 2+1 / 4\right)-1 / 4, \quad \widetilde{w}_{k}=\left(x_{k}-\rho_{1}\right) w_{k}\left(\rho_{2}, \rho_{1}, r_{1}, r_{2}\right) .
$$

2. For $r_{1}=\frac{N+2}{2}+\rho_{1}$ or $r_{1}=\frac{N+1}{2}-r_{2}$ with $N$ odd:

$$
x_{k}=(-1)^{k}\left(r_{1}-k / 2-1 / 4\right)-1 / 4, \quad \widetilde{w}_{k}=\left(x_{k}-\rho_{1}\right) w_{k}\left(-r_{1},-r_{2},-\rho_{1},-\rho_{2}\right) .
$$

3. For $r_{2}=\frac{N+2}{2}+\rho_{1}$ with $N$ odd:

$$
x_{k}=(-1)^{k}\left(r_{2}-k / 2-1 / 4\right)-1 / 4, \quad \widetilde{w}_{k}=\left(x_{k}-\rho_{1}\right) w_{k}\left(-r_{2},-r_{1},-\rho_{1},-\rho_{2}\right) .
$$

Proof. The proof follows from the above considerations.

Note that the normalization factor $\widetilde{h}_{n}$ appearing in (3.9) can easily be evaluated in terms of the product $\tau_{1} \tau_{2} \cdots \tau_{n}$. 
The complementary Bannai-Ito polynomials can be obtained from the Askey-Wilson polynomials upon taking the $q \rightarrow-1$ limit [21]. Consider the Askey-Wilson polynomials [13] $p_{n}(z ; a, b, c, d)$

$$
p_{n}(z ; a, b, c, d)=a^{-n}(a b, a c, a d ; q)_{n}{ }_{4} \phi_{3}\left(\begin{array}{c}
q^{-n}, a b c d q^{n-1}, a z, a z^{-1} \\
a b, a c, a d
\end{array} \mid q ; q\right),
$$

where $\phi$ denotes the basic generalized hypergeometric function [5]. These polynomials depend on the argument $x=\left(z+z^{-1}\right) / 2$ and on four complex parameters $a, b, c$ and $d$. They obey the recurrence relation $[13]$

$$
\alpha_{n} p_{n+1}(z)+\left(a+a^{-1}-\alpha_{n}-\gamma_{n}\right) p_{n}(z)+\gamma_{n} p_{n-1}(z)=\left(z+z^{-1}\right) p_{n}(z)
$$

where the coefficients are

$$
\begin{aligned}
& \alpha_{n}=\frac{\left(1-a b q^{n}\right)\left(1-a c q^{n}\right)\left(1-a d q^{n}\right)\left(1-a b c d q^{n-1}\right)}{a\left(1-a b c d q^{2 n-1}\right)\left(1-a b c d q^{2 n}\right)} \\
& \gamma_{n}=\frac{a\left(1-q^{n}\right)\left(1-b c q^{n-1}\right)\left(1-b d q^{n-1}\right)\left(1-c d q^{n-1}\right)}{\left(1-a b c d q^{2 n-2}\right)\left(1-a b c d q^{2 n-1}\right)} .
\end{aligned}
$$

To recover the CBI polynomials, we consider the parametrization

$$
\begin{array}{lll}
a=i e^{\epsilon\left(2 \rho_{1}+3 / 2\right)}, & b=-i e^{\epsilon\left(2 \rho_{2}+1 / 2\right)}, & c=i e^{\epsilon\left(-2 r_{2}+1 / 2\right),} \\
d=i e^{\epsilon\left(-2 r_{1}+1 / 2\right)}, & q=-e^{\epsilon}, & z=i e^{-2 \epsilon y} .
\end{array}
$$

It can be verified that the limit $q \rightarrow-1$ of the Askey-Wilson polynomials

$$
\lim _{q \rightarrow-1} p_{n}(z)=p_{n}^{*}(y)
$$

exists [21] and that $p_{n}^{*}(y)$ is a polynomial of degree $n$ in the variable $y$. Dividing the recurrence relation (3.17) by $1+q$ and taking the limit $\epsilon \rightarrow 0$, which amounts to taking $q \rightarrow-1$, one finds that the recurrence relation of the limit polynomials $p_{n}^{*}(y)$ is

$$
\alpha_{n}^{*} p_{n+1}^{*}(y)+(-1)^{n} \rho_{2} p_{n}^{*}(y)+\gamma_{n}^{*} p_{n-1}^{*}(y)=(y-1 / 4) p_{n}^{*}(y),
$$

where

$$
\begin{aligned}
& \alpha_{2 n}^{*}=-\frac{\left(n+\rho_{1}+\rho_{2}+1\right)(n+g+1)}{(2 n+g+1)}, \\
& \alpha_{2 n+1}^{*}=-\frac{\left(n+\rho_{1}-r_{1}+3 / 2\right)\left(n+\rho_{1}-r_{2}+3 / 2\right)}{(2 n+g+2)}, \\
& \gamma_{2 n}^{*}=\frac{n\left(n-r_{1}-r_{2}\right)}{(2 n+g+1)}, \\
& \gamma_{2 n+1}^{*}=\frac{\left(n+\rho_{2}-r_{1}+1 / 2\right)\left(n+\rho_{2}-r_{2}+1 / 2\right)}{(2 n+g+2)} .
\end{aligned}
$$

From (3.5) and (3.19), one has the identification

$$
\widehat{p_{n}^{*}}(y)=I_{n}(y-1 / 4),
$$

where $\widehat{p_{n}^{*}}$ are the monic version of the limit polynomials $p_{n}^{*}(y)$. Consequently, the CBI polynomial correspond to a $q \rightarrow-1$ limit of the Askey-Wilson polynomials, up to a shift in argument. This property will be used in the next section to construct a Dunkl operator that has the CBI polynomials as eigenfunctions. 


\section{Bispectrality of CBI polynomials}

In this section, we obtain a family of second order Dunkl shift operators for which the complementary Bannai-Ito polynomials are eigenfunctions with eigenvalues quadratic in $n$. This family will be constructed from a limit of a quadratic combination of the Askey-Wilson $q$-difference operator. We shall refer to these operators as the defining operators of the CBI polynomials.

Consider the Askey-Wilson polynomials $p_{n}(x)$ defined by (3.16). They obey the $q$-difference equation [13]

$$
\left(\Omega(z) E_{z, q}+\Omega\left(z^{-1}\right) E_{z, q^{-1}}-\left(\Omega(z)+\Omega\left(z^{-1}\right)\right) \mathbb{I}\right) p_{n}(z)=\Lambda_{n} p_{n}(z)
$$

where $E_{z, q} f(z)=f(q z)$ is the $q$-shift operator and $\mathbb{I}$ denotes the identity. The l.h.s. of (4.1) is the Askey-Wilson operator. The eigenvalues take the form

$$
\Lambda_{n}=\left(q^{-n}-1\right)\left(1-a b c d q^{n-1}\right)
$$

and the coefficient $\Omega(z)$ is given by

$$
\Omega(z)=\frac{(1-a z)(1-b z)(1-c z)(1-d z)}{\left(1-z^{2}\right)\left(1-q z^{2}\right)} .
$$

We now consider the limiting form of the $q$-difference equation (4.1) when $q \rightarrow-1$. As done previously, we choose the parametrization (3.18), which correspond to the CBI polynomials. We already showed that the Askey-Wilson polynomials $p_{n}(z)$ become the complementary BannaiIto polynomials $p_{n}^{*}(y)$. In the limit $q \rightarrow-1$, the $q$-shift operation $p_{n}(z) \rightarrow p_{n}(q z)$ becomes $p_{n}^{*}(y) \rightarrow p_{n}^{*}(-y+1 / 2)$ while $p_{n}(z) \rightarrow p_{n}\left(q^{-1} z\right)$ is reduced to $p_{n}^{*}(y) \rightarrow p_{n}^{*}(-y-1 / 2)$.

It is natural to expect that in the limit $q \rightarrow-1$, the equation (4.1) will yield a defining operator for the CBI polynomials. However a direct computation shows that the limit $\epsilon \rightarrow 0$ of the equation (4.1) with the parametrization (3.18) does not exist. It is hence impossible to find the desired operator for the CBI polynomials directly from a limiting procedure on equation (4.1). Nevertheless, it is possible to work around this difficulty by choosing an appropriate quadratic combination of the Askey-Wilson operator that survives the limit $q \rightarrow-1$. A similar procedure was used in [20] to establish the bispectrality of the dual -1 Hahn polynomials.

Let $\mathcal{O}$ denote the Askey-Wilson operator

$$
\mathcal{O}=\Omega(z) E_{z, q}+\Omega\left(z^{-1}\right) E_{z, q^{-1}}-\left(\Omega(z)+\Omega\left(z^{-1}\right)\right) \mathbb{I}
$$

which acts on the space of functions $f(z)$ of argument $z$. We consider the following quadratic combination

$$
\mathcal{T}=c^{(2)} \mathcal{O}^{2}+c^{(1)} \mathcal{O}
$$

with

$$
c^{(2)}=\frac{1}{16(1+q)^{2}}, \quad c^{(1)}=\frac{1}{4}\left(\frac{1}{(q+1)^{2}}-\frac{g+1}{q+1}\right),
$$

where $g=\rho_{1}+\rho_{2}-r_{1}-r_{2}$. Since the operator $\mathcal{O}$ acts diagonally on the Askey-Wilson polynomials, we have

$$
\mathcal{T} p_{n}(z)=\left(c^{(2)} \Lambda_{n}^{2}+c^{(1)} \Lambda_{n}\right) p_{n}(x)
$$


Upon taking the limit $\epsilon \rightarrow 0$ with the parametrization (3.18), the relation (4.2) becomes

$$
\begin{gathered}
\Phi_{1}(y) p_{n}^{*}(y+1)+\left\{\Phi_{5}(y)-\Phi_{2}(y)-\Phi_{3}(y)\right\} p_{n}^{*}(1 / 2-y)+\left\{\Phi_{3}(y)-\Phi_{4}(y)-\Phi_{5}(y)\right\} p_{n}^{*}(y) \\
+\left\{\Phi_{4}(y)-\Phi_{1}(y)\right\} p_{n}^{*}(-y-1 / 2)+\Phi_{2}(y) p_{n}^{*}(y-1)=\kappa_{n} p_{n}^{*}(y)
\end{gathered}
$$

where the eigenvalues are

$$
\kappa_{2 n}=n^{2}+(g+1) n, \quad \kappa_{2 n+1}=n^{2}+(g+2) n+g^{2}+2 g+5 / 4 .
$$

The coefficients $\Phi_{i}(y)$ are given by

$$
\begin{aligned}
& \Phi_{1}(y)=\frac{\left(y+\rho_{1}+3 / 4\right)\left(y+\rho_{2}+3 / 4\right)\left(y-r_{1}+1 / 4\right)\left(y-r_{2}+1 / 4\right)}{4(y+1 / 4)(y+3 / 4)}, \\
& \Phi_{2}(y)=\frac{\left(y-\rho_{1}-5 / 4\right)\left(y-\rho_{2}-1 / 4\right)\left(y+r_{1}-3 / 4\right)\left(y+r_{2}-3 / 4\right)}{4(y-1 / 4)(y-3 / 4)}, \\
& \Phi_{3}(y)=\frac{\left(y+\rho_{1}+3 / 4\right)\left(y-\rho_{2}-1 / 4\right)\left(y-r_{1}+1 / 4\right)\left(y-r_{2}+1 / 4\right)}{4(y-1 / 4)(y+1 / 4)}, \\
& \Phi_{4}(y)=\frac{\left(y+\rho_{1}+3 / 4\right)\left(y+\rho_{2}-1 / 4\right)\left(y-r_{1}+1 / 4\right)\left(y-r_{2}+1 / 4\right)}{4(y-1 / 4)(y+1 / 4)}, \\
& \Phi_{5}(y)=\frac{\left(y-\rho_{2}-1 / 4\right)}{4(y-1 / 4)}\left\{2 y^{2}-y+\nu\right\},
\end{aligned}
$$

where $\nu$ takes the form

$$
\nu=r_{1}+r_{2}+2 r_{1} r_{2}-2 \rho_{1}-2\left(r_{1}+r_{2}\right) \rho_{1}-4 \rho_{2}+1 / 8-2 g^{2} .
$$

By the identification (3.20), the relation (4.3) gives the complementary Bannai-Ito polynomials $I_{n}(x)$ as eigenfunctions of a second order Dunkl shift operator, hence establishing their bispectrality property. In operator form, the equation (4.3) may be rewritten as

$$
\mathcal{H} I_{n}(y-1 / 4)=\kappa_{n} I_{n}(y-1 / 4),
$$

where $\mathcal{H}$ has the expression

$$
\mathcal{H}=\Phi_{1} T^{1}+\left(\Phi_{4}-\Phi_{1}\right) T^{1 / 2} R+\left(\Phi_{3}-\Phi_{4}-\Phi_{5}\right) \mathbb{I}+\left(\Phi_{5}-\Phi_{2}-\Phi_{3}\right) T^{-1 / 2} R+\Phi_{2} T^{-1},
$$

where $T^{h} f(y)=f(y+h)$ and $R f(y)=f(-y)$. Upon applying the unitary transformation

$$
\widetilde{\mathcal{H}}=T^{1 / 4} \mathcal{H} T^{-1 / 4}
$$

on the operator $\mathcal{H}$ and changing the variable from $y$ to $x$, the eigenvalue equation (4.3) for the CBI polynomials becomes

$$
\widetilde{\mathcal{H}} I_{n}(x)=\kappa_{n} I_{n}(x)
$$

where we have

$$
\widetilde{\mathcal{H}}=\widetilde{\Phi}_{1} T^{+}+\left(\widetilde{\Phi}_{4}-\widetilde{\Phi}_{1}\right) T^{+} R+\left(\widetilde{\Phi}_{3}-\widetilde{\Phi}_{4}-\widetilde{\Phi}_{5}\right) \mathbb{I}+\left(\widetilde{\Phi}_{5}-\widetilde{\Phi}_{2}-\widetilde{\Phi}_{3}\right) R+\widetilde{\Phi}_{2} T^{-},
$$

with $T^{+}=T^{1}$ and $T^{-}=T^{-}$the usual shift operators in $x$. The coefficients now have the expression

$$
\widetilde{\Phi}_{i}=\Phi_{i}(x+1 / 4) .
$$


We now turn to the study of the uniqueness of the operator $\mathcal{H}$ which defines the eigenvalue equation of the complementary Bannai-Ito polynomials (apart from trivial affine transformations). Quite strikingly, a one-parameter family of such operators can be constructed. This peculiarity is due to the presence of a "hidden" symmetry in the CBI polynomials. To see this, we recall the relation (3.14) for the CBI polynomials

$$
I_{2 n}=R_{n}\left(x^{2}\right), \quad I_{2 n+1}=\left(x-\rho_{2}\right) Q_{n}\left(x^{2}\right),
$$

where $R_{n}\left(x^{2}\right)$ and $Q_{n}\left(x^{2}\right)$ are monic polynomials of degree $n$. From the above relation, it is easily seen that

$$
I_{2 n}(-x)=I_{2 n}(x), \quad \text { and } \quad I_{2 n+1}(-x)=\frac{\left(x+\rho_{2}\right)}{\left(\rho_{2}-x\right)} I_{2 n+1}(x) .
$$

The above equations are equivalent to the following non-trivial "hidden" eigenvalue equation for the CBI polynomials

$$
\frac{\left(\rho_{2}-x\right)}{2 x}\left(I_{n}(-x)-I_{n}(x)\right)=\mu_{n} I_{n}(x)
$$

where $\mu_{2 n}=0$ and $\mu_{2 n+1}=1$. In operator form, we write

$$
\frac{\left(x-\rho_{2}\right)}{2 x}(\mathbb{I}-R) I_{n}(x)=\mathcal{U} I_{n}(x)=\mu_{n} I_{n}(x) .
$$

The equation (4.5) indicates that adding $\alpha \mathcal{U}$ to the operator (4.4) will give another eigenvalue equation for the complementary Bannai-Ito polynomials. The modified operator

$$
\widetilde{\mathcal{H}}^{\prime}=\widetilde{\mathcal{H}}+\alpha \mathcal{U},
$$

will have the same spectrum as $\widetilde{\mathcal{H}}$ in the even sector; in the odd sector, the eigenvalues will differ by the constant parameter $\alpha$.

For definiteness and future reference, let us now collect the preceding results in the following theorem.

Theorem 1. Let $\mathcal{D}_{0}$ be the second order Dunkl shift operator acting on the space of functions $f(x)$ of argument $x$

$$
\mathcal{D}_{0}=A(x) T^{+}+B(x) T^{-}+C(x) R+D(x) T^{+} R-(A(x)+B(x)+C(x)+D(x)) \mathbb{I},
$$

where $T^{ \pm} f(x)=f(x \pm 1)$ and $R f(x)=f(-x)$, with the coefficients

$$
\begin{aligned}
& A(x)=\frac{\left(x+\rho_{1}+1\right)\left(x+\rho_{2}+1\right)\left(2 x-2 r_{1}+1\right)\left(2 x-2 r_{2}+1\right)}{8(x+1)(2 x+1)}, \\
& B(x)=\frac{\left(x-\rho_{2}\right)\left(x-\rho_{1}-1\right)\left(2 x+2 r_{1}-1\right)\left(2 x+2 r_{2}-1\right)}{8 x(2 x-1)}, \\
& C(x)=\frac{\left(x-\rho_{2}\right)\left(4 x^{2}+\omega\right)}{8 x}-\frac{\left(x-\rho_{2}\right)\left(x+\rho_{1}+1\right)\left(2 x-2 r_{1}+1\right)\left(2 x-2 r_{2}+1\right)}{8 x(2 x+1)}-B(x), \\
& D(x)=\frac{\rho_{2}\left(x+\rho_{1}+1\right)\left(2 x-2 r_{1}+1\right)\left(2 x-2 r_{2}+1\right)}{8 x(x+1)(2 x+1)},
\end{aligned}
$$

and with

$$
\omega=4 \rho_{1}-4\left(r_{1}+r_{2}\right) \rho_{1}+4 r_{1} r_{2}-6\left(r_{1}+r_{2}\right)+5 .
$$


Furthermore, let $\alpha \in \mathbb{C}$ be a complex number and denote the monic complementary Bannai-Ito polynomials by $I_{n}(x)$. Then the following eigenvalue equation is satisfied:

$$
\mathcal{D}_{\alpha} I_{n}(x)=\Lambda_{n}^{(\alpha)} I_{n}(x),
$$

where the eigenvalues are

$$
\Lambda_{2 n}^{(\alpha)}=n^{2}+(g+1) n, \quad \Lambda_{2 n+1}^{(\alpha)}=n^{2}+(g+2) n+\alpha,
$$

and where we have defined

$$
\mathcal{D}_{\alpha}=\mathcal{D}_{0}+\alpha \frac{\left(x-\rho_{2}\right)}{2 x}(\mathbb{I}-R) .
$$

Proof. The result follows from the above considerations.

We now discuss the CBI polynomials in the context of the Leonard duality. A family of orthogonal polynomials $\mathcal{P}_{n}(x)$ is said to possess the Leonard duality property if it satisfies both a three-term recurrence relation with respect to $n$ and a three-term difference equation of the form

$$
\theta\left(x_{k}\right) \mathcal{P}_{n}\left(x_{k+1}\right)+\nu\left(x_{k}\right) \mathcal{P}_{n}\left(x_{k}\right)+\mu\left(x_{k}\right) \mathcal{P}_{n}\left(x_{k-1}\right)=\vartheta_{n} \mathcal{P}_{n}\left(x_{k}\right),
$$

on a discrete set of points $x_{k}, k \in \mathbb{Z}$. The classification of the polynomials with this property was first accomplished by Leonard in [14]; his theorem was later generalized to include infinitedimensional grids by Bannai and Ito [1]. It turns out that the complementary Bannai-Ito polynomials lie beyond the scope of the Leonard duality. Indeed, the operators $\mathcal{D}_{\alpha}$ can be used to show that the CBI polynomials obey a five-term difference equation on an infinite-dimensional grid. This result is obtained in the following way.

First consider the grid $x_{k}$ defined by

$$
x_{k}=(-1)^{k}(k / 2+h+1 / 4)-1 / 4, \quad k \in \mathbb{Z},
$$

where $h$ is an arbitrary real parameter. It is easily seen that the grid (4.10) is preserved by the operators appearing in (4.6). Explicitly, we have

$$
\begin{aligned}
& T^{+} x_{k}= \begin{cases}x_{k+2}, & k \text { even, } \\
x_{k-2}, & k \text { odd },\end{cases} \\
& R x_{k}= \begin{cases}x_{k-1}, & k \text { even, } \\
x_{k+1}, & k \text { odd },\end{cases}
\end{aligned}
$$

Referring to $\mathcal{D}_{0}$, one finds the following five-term difference equation for the CBI polynomials:

$$
\begin{aligned}
& u\left(x_{k}\right) I_{n}\left(x_{k+2}\right)+v\left(x_{k}\right) I_{n}\left(x_{k+1}\right)+m\left(x_{k}\right) I_{n}\left(x_{k}\right) \\
& \quad+t\left(x_{k}\right) I_{n}\left(x_{k-1}\right)+r\left(x_{k}\right) I_{m}\left(x_{k-2}\right)=\Lambda_{n}^{(0)} I_{n}\left(x_{k}\right)
\end{aligned}
$$

where we have

$$
\begin{aligned}
& u\left(x_{k}\right)=\left\{\begin{array}{lll}
A\left(x_{k}\right), & k \text { even, } \\
B\left(x_{k}\right), & k \text { odd, }
\end{array} \quad v\left(x_{k}\right)= \begin{cases}D\left(x_{k}\right), & k \text { even, } \\
C\left(x_{k}\right), & k \text { odd },\end{cases} \right. \\
& t\left(x_{k}\right)=\left\{\begin{array}{lll}
C\left(x_{k}\right), & k \text { even, } \\
D\left(x_{k}\right), & k \text { odd, }
\end{array} \quad r\left(x_{k}\right)= \begin{cases}B\left(x_{k}\right), & k \text { even, } \\
A\left(x_{k}\right), & k \text { odd },\end{cases} \right.
\end{aligned}
$$

and $-m\left(x_{k}\right)=u\left(x_{k}\right)+v\left(x_{k}\right)+t\left(x_{k}\right)+r\left(x_{k}\right)$. A similar relation can be found for any value of $\alpha$. Moreover, it is possible to obtain another 5 -term difference equation by considering the alternative grid

$$
\widetilde{x}_{k}=(-1)^{k}(h-k / 2-1 / 4)-1 / 4, \quad k \in \mathbb{Z},
$$

and proceeding along the same lines. 


\section{The CBI algebra}

The Bannai-Ito polynomials have as an underlying algebraic structure the so-called BI algebra [21], which corresponds to a $q \rightarrow-1$ limit of the Askey-Wilson $(A W(3))$ algebra [29]. The algebra $A W(3)$ and the related concept of Leonard pairs [18, 19, 23], describe polynomials which possess the Leonard duality. In this section, we obtain the algebraic structure that encodes the properties of the CBI polynomials.

We begin by a formal definition of the CBI algebra.

Definition 1. The complementary Bannai-Ito (CBI) algebra is generated by the elements $\kappa_{1}$, $\kappa_{2}, \kappa_{3}$ and the involution $r$ satisfying the relations

$$
\begin{aligned}
& {\left[\kappa_{1}, r\right]=0, \quad\left\{\kappa_{2}, r\right\}=2 \delta_{3}, \quad\left\{\kappa_{3}, r\right\}=0, \quad\left[\kappa_{1}, \kappa_{2}\right]=\kappa_{3},} \\
& {\left[\kappa_{1}, \kappa_{3}\right]=\frac{1}{2}\left\{\kappa_{1}, \kappa_{2}\right\}-\delta_{2} \kappa_{3} r-\delta_{3} \kappa_{1} r+\delta_{1} \kappa_{2}-\delta_{1} \delta_{3} r, \quad r^{2}=\mathbb{I},} \\
& {\left[\kappa_{3}, \kappa_{2}\right]=\frac{1}{2} \kappa_{2}^{2}+\delta_{2} \kappa_{2}^{2} r+2 \delta_{3} \kappa_{1} r+2 \delta_{3} \kappa_{3} r+\kappa_{1}+\delta_{4} r+\delta_{5},}
\end{aligned}
$$

where $[x, y]=x y-y x$ and $\{x, y\}=x y+y x$. The CBI algebra (5.1) admits the Casimir operator

$$
\begin{aligned}
Q= & \frac{1}{2}\left\{\kappa_{2}^{2}, \kappa_{1}\right\}-\frac{\delta_{2}}{2} \kappa_{2}^{2} r+\kappa_{1}^{2}-\kappa_{3}^{2}+\left(\delta_{1}-1 / 4\right) \kappa_{2}^{2} \\
& +\left(\delta_{3}-\delta_{2}\right) \kappa_{1} r+2 \delta_{5} \kappa_{1}+\left(\delta_{1} \delta_{3}-\delta_{2} \delta_{5}\right) r,
\end{aligned}
$$

which commutes with all the generators.

We define the operators

$$
K_{1}=\mathcal{D}_{\alpha}, \quad K_{2}=x,
$$

where $K_{2}$ is the operator multiplication by $x$ and where $\mathcal{D}_{\alpha}$ is as given by (4.9). We introduce the involution [7]

$$
P=R+\frac{\rho_{2}}{x}(\mathbb{I}-R) .
$$

It is easily seen that $P^{2}=\mathbb{I}$. Finally, we define a fourth operator $K_{3}$ as follows:

$$
K_{3}=A(x) T^{+}-B(x) T^{-}+\left[\alpha\left(x-\rho_{2}\right)-2 x C(x)\right] R-(1+2 x) D(x) T^{+} R .
$$

A direct computation shows that the operators $K_{1}, K_{2}$ and $K_{3}$, together with the involution $P$, realize the CBI algebra (5.1) under the identifications

$$
K_{1}=\kappa_{1}, \quad K_{2}=\kappa_{2}, \quad K_{3}=\kappa_{3}, \quad P=r .
$$

The structure constants take the form

$$
\begin{aligned}
& \delta_{1}=\alpha(g-\alpha+1), \quad \delta_{2}=g-2 \alpha+3 / 2, \quad \delta_{3}=\rho_{2}, \quad \delta_{5}=\alpha\left(\rho_{2}-1 / 2\right)+\omega / 8, \\
& \delta_{4}=\alpha\left(2 \rho_{2}^{2}-\rho_{2}+1 / 2\right)+\rho_{2} \omega / 4+\left(8 \rho_{1} r_{1} r_{2}+4 r_{1} r_{2}-2 \rho_{1}+2 r_{1}+2 r_{2}-3\right) / 8 .
\end{aligned}
$$

It is worth pointing out that even though the BI and CBI polynomials can be obtained from one another by a Christoffel (resp. Geronimus) transformation and that they can both be obtained from the Askey-Wilson polynomials by very similar $q \rightarrow-1$ limits, their underlying algebraic structure are very dissimilar [21]. In the realization (5.3)-(5.5) the Casimir operator (5.2) acts a multiple of the identity

$$
Q f(x)=q f(x),
$$

where $q$ is a complicated function of the five parameters $\rho_{1}, \rho_{2}, r_{1}, r_{2}$ and $\alpha$. 
The realization (5.3)-(5.5) can be used to obtain irreducible representations of the algebra (5.1) in two "dual" bases. In the first basis $\left\{v_{n}, n \in \mathbb{N}\right\}$, the operator $\kappa_{1}$ is diagonal:

$$
\kappa_{1} v_{n}=\Lambda_{n}^{(\alpha)} v_{n}
$$

where $\Lambda_{n}^{(\alpha)}$ is given by (4.8). Since $\kappa_{1}$ and $r$ commute, the operator $r$ can also be taken diagonal in this representation. Since $r^{2}=\mathbb{I}$, one finds

$$
r v_{n}=\epsilon(-1)^{n} v_{n}
$$

where $\epsilon= \pm 1$ is a representation parameter. Given the fact that the representation parameter $\epsilon$ is only a global multiplication factor of $r$, one can choose $\epsilon=1$ without loss of generality. Because $r$ is diagonal in the basis $v_{n}$, the matrix elements of $\kappa_{2}$ in the basis $v_{n}$ can be calculated in a way similar to the one employed to obtain the representations of the Hahn algebra [8], with additional parity requirements. It is straightforward to show that in the basis $v_{n}$, upon choosing the initial condition $a_{0}=0$, the operator $\kappa_{2}$ is tridiagonal with the action

$$
\kappa_{2} v_{n}=a_{n+1} v_{n+1}+b_{n} v_{n}+a_{n} v_{n-1}
$$

where we have

$$
a_{n}=\sqrt{\tau_{n}}, \quad b_{n}=(-1)^{n} \rho_{2},
$$

with $\tau_{n}$ given as in (3.5). We thus have the following result.

Proposition 2. Let $V$ be the infinite-dimensional $\mathbb{C}$-vector space spanned by the basis vectors $\left\{v_{n} \mid n \in \mathbb{N}\right\}$ endowed with the actions

$$
\begin{aligned}
& \kappa_{1} v_{n}=\Lambda_{n}^{(\alpha)} v_{n}, \quad r v_{n}=(-1)^{n} v_{n}, \\
& \kappa_{2} v_{n}=\sqrt{\tau_{n+1}} v_{n+1}+(-1)^{n} \rho_{2} v_{n}+\sqrt{\tau_{n}} v_{n-1}, \\
& \kappa_{3} v_{n}=\left(\Lambda_{n+1}^{(\alpha)}-\Lambda_{n}^{(\alpha)}\right) \sqrt{\tau_{n+1}} v_{n+1}-\left(\Lambda_{n}^{(\alpha)}-\Lambda_{n-1}^{(\alpha)}\right) \sqrt{\tau_{n}} v_{n-1},
\end{aligned}
$$

where $\Lambda_{n}^{(\alpha)}$ and $\tau_{n}$ are given by (4.8) and (3.5), respectively. Then $V$ is a module for the $C B I$ algebra (5.1) with structure constants taking the values (5.6). The module is irreducible if none of the truncation conditions (3.8) and (3.10) are satisfied.

Proof. The above considerations show that $V$ is indeed a CBI-module. The irreducibility stems from the fact that if the none of the truncation conditions (3.8) and (3.10) are satisfied, then $\tau_{n}$ is never zero.

Corollary. If one of the truncation conditions (3.8) or (3.10) is satisfied, then $V$ is no longer irreducible. One can restrict to the subspace spanned by the basis vectors $\left\{v_{n} \mid n=0, \ldots, N\right\}$ and obtain a $(N+1)$-dimensional irreducible CBI-module.

Thus the CBI algebra admits infinite-dimensional representations where $\kappa_{1}, r$ are diagonal and $\kappa_{2}$ is tridiagonal with matrix elements (5.7). Is is readily checked that

$$
P I_{n}(x)=(-1)^{n} I_{n}(x)
$$

and hence it is clear that the basis vectors $v_{n}$ correspond to the CBI polynomials themselves

$$
v_{n}=I_{n}(x)
$$


Alternatively, we can consider the "dual" basis $\left\{\psi_{k}, k \in \mathbb{Z}\right\}$, in which the operator $\kappa_{2}$ is diagonal

$$
\kappa_{2} \psi_{k}=\vartheta_{k} \psi_{k}
$$

with the Bannai-Ito spectrum

$$
\vartheta_{k}=(-1)^{k}(k / 2+t+1 / 4)-1 / 4,
$$

where $t$ an arbitrary real constant. In this basis, the involution $r$ cannot be diagonal. Let $A_{\ell k}$ be the matrix elements of $r$ in the basis $\psi_{k}$. We have

$$
r \psi_{k}=\sum_{\ell} A_{\ell k} \psi_{\ell}
$$

Written in the basis $\psi_{k}$, the anticommutation relation $\left\{\kappa_{2}, r\right\}=2 \rho_{2}$ has the simple form

$$
\sum_{\ell} A_{\ell, k}\left\{\vartheta_{\ell}+\vartheta_{k}\right\} \psi_{\ell}=2 \rho_{2} \psi_{k}
$$

For $\ell=k$, this yields

$$
A_{2 k, 2 k}=\frac{\rho_{2}}{k+t}, \quad A_{2 k+1,2 k+1}=-\frac{\rho_{2}}{k+t+1} .
$$

When $\ell \neq k$, the equation (5.9) reduces to

$$
A_{k, \ell}\left\{\vartheta_{k}+\vartheta_{\ell}\right\}=0 \text {. }
$$

From the definition (5.8) of the eigenvalues $\vartheta_{k}$, one notes that

$$
\vartheta_{2 k+1}+\vartheta_{2 k+2}=0 \text {. }
$$

It follows from (5.10) that in the basis $\psi_{k}$, the operator $r$ is block diagonal with all blocks $2 \times 2$. Upon demanding that the other commutation relations of (5.1) be satisfied, it can be shown [7] that in this basis, the operator $\kappa_{1}$ becomes 5-diagonal. This result is expected since the CBI polynomials obey a 5 -term difference equation of the form (4.11) on the Bannai-Ito grid.

We have obtained that the CBI polynomials are eigenfunctions of a one-parameter family of operators of the form (4.9) and that two operators $\mathcal{D}_{\alpha}, \mathcal{D}_{\beta}$ of this family are related by the "hidden" symmetry operator of the CBI polynomials given by (4.5). In the CBI algebra, the transformation $\mathcal{D}_{\alpha} \rightarrow \mathcal{D}_{\alpha+\beta}$ is equivalent to defining

$$
\widetilde{K}_{1}=K_{1}+\frac{\beta}{2}(\mathbb{I}-P),
$$

while leaving $K_{2}$ and $P$ unchanged. The operator $K_{3}$ is transformed to

$$
\widetilde{K}_{3}=K_{3}-\beta P K_{2}+\beta \delta_{3} .
$$

Upon using $\widetilde{K}_{2}=K_{2}$, one finds that the algebra becomes

$$
\begin{aligned}
& {\left[\widetilde{K}_{1}, P\right]=0, \quad\left\{\widetilde{K}_{2}, P\right\}=2 \widetilde{\delta}_{3}, \quad\left\{\widetilde{K}_{3}, P\right\}=0, \quad\left[\widetilde{K}_{1}, \widetilde{K}_{2}\right]=\widetilde{K}_{3},} \\
& {\left[\widetilde{K}_{1}, \widetilde{K}_{3}\right]=\frac{1}{2}\left\{\widetilde{K}_{1}, \widetilde{K}_{2}\right\}-\widetilde{\delta}_{2} \widetilde{K}_{3} P-\widetilde{\delta}_{3} \widetilde{K}_{1} P+\widetilde{\delta}_{1} \widetilde{K}_{2}-\widetilde{\delta}_{1} \widetilde{\delta}_{3} P,} \\
& {\left[\widetilde{K}_{3}, \widetilde{K}_{2}\right]=\frac{1}{2} \widetilde{K}_{2}^{2}+\widetilde{\delta}_{2} \widetilde{K}_{2}^{2} P+2 \widetilde{\delta}_{3} \widetilde{K}_{1} P+2 \widetilde{\delta}_{3} \widetilde{K}_{3} P+\widetilde{K}_{1}+\widetilde{\delta}_{4} P+\widetilde{\delta}_{5},}
\end{aligned}
$$

with the structures constants

$$
\begin{aligned}
& \widetilde{\delta}_{1}=\delta_{1}+\beta\left(\delta_{2}-1 / 2\right), \quad \widetilde{\delta}_{2}=\delta_{2}-2 \beta, \quad \widetilde{\delta}_{3}=\delta_{3}, \\
& \widetilde{\delta}_{4}=\delta_{4}+\beta\left(2 \delta_{3}^{2}-\delta_{3}+1 / 2\right), \quad \widetilde{\delta}_{5}=\delta_{5}+\beta\left(\delta_{3}-1 / 2\right) .
\end{aligned}
$$

It is thus seen that the transformation (5.11) leaves the general form of the CBI algebra (5.1) unaffected and corresponds only to a change in the structure parameters. 


\section{Three OPs families related to the CBI polynomials}

In this section, we exhibit the relationship between the complementary Bannai-Ito polynomials and three other families of orthogonal polynomials: the recently discovered dual -1 Hahn [20, 27] and para-Krawtchouk polynomials [28] and the classical symmetric Hahn polynomials.

\subsection{Dual -1 Hahn polynomials}

The dual -1 Hahn polynomials have been introduced in [20] as $q=-1$ limits of the dual $q$-Hahn polynomials. They have appeared in the context of perfect state transfer in spin chains [27] and also as the Clebsch-Gordan coefficients of the $s l_{-1}(2)$ algebra in [6, 22]. Moreover, the -1 Hahn polynomials have occurred, in their symmetric form, as wavefunctions for finite parabosonic oscillator models $[9,10]$. These polynomials ${ }^{1}$, denoted $Q_{n}(x)$, can be obtained from the CBI polynomials through the limit $\rho_{1} \rightarrow \infty$.

Taking the limit $\rho_{1} \rightarrow \infty$ in the (3.5), one obtains the recurrence relation of the monic dual -1 Hahn polynomials

$$
Q_{n+1}(x)+(-1)^{n} \rho_{2} Q_{n}(x)+\sigma_{n} Q_{n-1}(x)=x Q_{n}(x),
$$

where $r_{n}$ has the expression

$$
\sigma_{2 n}=-n\left(n-r_{1}-r_{2}\right), \quad \sigma_{2 n+1}=-\left(n+\rho_{2}-r_{1}+1 / 2\right)\left(n+\rho_{2}-r_{2}+1 / 2\right) .
$$

The polynomials $Q_{n}(x)$ have the hypergeometric representation

$$
\begin{aligned}
& Q_{2 n}(x)=\xi_{2 n 3} F_{2}\left[\begin{array}{c}
-n, \rho_{2}+x, \rho_{2}-x \\
\rho_{2}-r_{1}+1 / 2, \rho_{2}-r_{2}+1 / 2
\end{array} ; 1\right], \\
& Q_{2 n+1}(x)=\xi_{2 n+1}\left(x-\rho_{2}\right){ }_{3} F_{2}\left[\begin{array}{c}
-n, \rho_{2}+x+1, \rho_{2}-x+1 \\
\rho_{2}-r_{1}+3 / 2, \rho_{2}-r_{2}+3 / 2
\end{array} ; 1\right],
\end{aligned}
$$

with normalization coefficients

$$
\xi_{2 n}=\left(\rho_{2}-r_{1}+1 / 2\right)_{n}\left(\rho_{2}-r_{2}+1 / 2\right)_{n}, \quad \xi_{2 n+1}=\left(\rho_{2}-r_{1}+3 / 2\right)_{n}\left(\rho_{2}-r_{2}+3 / 2\right)_{n} .
$$

These formulas are obtained from (3.15) in the same limit. Dividing (4.9) by $\rho_{1}$ and taking the limit $\rho_{1} \rightarrow \infty$, one finds that the polynomials $Q_{n}(x)$ satisfy the eigenvalue equation

$$
\mathcal{E}^{(\alpha)} Q_{n}(x)=\nu_{n}^{(\alpha)} Q_{n}(x),
$$

with eigenvalues

$$
\nu_{2 n}^{(\alpha)}=n, \quad \nu_{2 n+1}^{(\alpha)}=n+\alpha .
$$

The operator $\mathcal{E}^{(\alpha)}$ is found to be

$$
\mathcal{E}^{(\alpha)}=\mathcal{E}^{(0)}+\alpha \frac{\left(x-\rho_{2}\right)}{2 x}(\mathbb{I}-R),
$$

where

$$
\mathcal{E}^{(0)}=I(x) T^{+}+J(x) T^{-}+K(x) R+L(x) T^{+} R-(I(x)+J(x)+K(x)+L(x)) \mathbb{I} .
$$

\footnotetext{
${ }^{1}$ To recover the formulas found in [20], a re-parametrization is necessary.
} 
The coefficients are given by

$$
\begin{aligned}
& I(x)=\frac{\left(x+\rho_{2}+1\right)\left(2 x-2 r_{1}+1\right)\left(2 x-2 r_{2}+1\right)}{8(x+1)(2 x+1)}, \\
& J(x)=\frac{\left(\rho_{2}-x\right)\left(2 x+2 r_{1}-1\right)\left(2 x+2 r_{2}-1\right)}{8 x(2 x-1)}, \\
& K(x)=\frac{\left(x-\rho_{2}\right)\left(4 x^{2}+4 r_{1} r_{2}-1\right)}{4 x\left(4 x^{2}-1\right)} \\
& L(x)=\frac{\rho_{2}\left(2 x-2 r_{1}+1\right)\left(2 x-2 r_{2}+1\right)}{8 x(x+1)(2 x+1)} .
\end{aligned}
$$

Lastly, it is seen that in the limit $\rho_{1} \rightarrow \infty$, the CBI algebra becomes

$$
\begin{aligned}
& {\left[\kappa_{1}, r\right]=0, \quad\left\{\kappa_{2}, r\right\}=2 \gamma_{3}, \quad\left\{\kappa_{3}, r\right\}=0, \quad\left[\kappa_{1}, \kappa_{2}\right]=\kappa_{3},} \\
& {\left[\kappa_{1}, \kappa_{3}\right]=\gamma_{1} \kappa_{2}-\gamma_{1} \gamma_{3} r-\gamma_{2} \kappa_{3} r, \quad\left[\kappa_{3}, \kappa_{2}\right]=\gamma_{2} \kappa_{2}^{2} r+2 \gamma_{3} \kappa_{1} r+2 \gamma_{3} \kappa_{3} r+\kappa_{1}+\gamma_{4} r+\gamma_{5},}
\end{aligned}
$$

where we have identified $\kappa_{1}=\mathcal{E}^{(\alpha)}, \kappa_{2}=x$ and $P=r$ with $P$ given by (5.4). The structure parameters have the expression

$$
\begin{aligned}
\gamma_{1} & =\alpha(1-\alpha), \quad \gamma_{2}=1-2 \alpha, \quad \gamma_{3}=\rho_{2}, \\
\gamma_{4} & =\alpha\left(2 \rho_{2}^{2}-\rho_{2}+1 / 2\right)+\rho_{2}\left(1-r_{2}-r_{1}\right)+r_{1} r_{2}-1 / 4, \\
\gamma_{5} & =\left(2 \alpha \rho_{2}-\alpha-r_{1}-r_{2}+1\right) / 2 .
\end{aligned}
$$

Other properties of the polynomials $Q_{n}(x)$ can be obtained directly using the limiting procedure.

\subsection{The symmetric Hahn polynomials}

It is possible to relate the CBI polynomials to the symmetric Hahn polynomials $[12,13]$ through a direct identification of the CBI parameters. This identification can be performed in three different ways by examining the cases for which the defining operator $\mathcal{D}_{\alpha}$ (4.9) of the CBI polynomials reduces to a classical three-term difference operator involving only the discrete shifts $T^{+}, T^{-}$and the identity operator $\mathbb{I}$.

We consider the operator $\mathcal{D}_{\alpha}$ in (4.9) with the following parameter identification:

$$
\rho_{1}=-\frac{1}{2}, \quad \rho_{2}=0, \quad \alpha=\frac{1}{2}\left(1-r_{1}-r_{2}\right) .
$$

With these values of the parameters, the eigenvalue equation (4.7) reduces to

$$
B(x) I_{n}(x+1)-(B(x)+D(x)) I_{n}(x)+D(x) I_{n}(x-1)=\lambda_{n} I_{n}(x),
$$

with coefficients

$$
B(x)=\left(x-r_{1}+1 / 2\right)\left(x-r_{2}+1 / 2\right), \quad D(x)=\left(x+r_{1}-1 / 2\right)\left(x+r_{2}-1 / 2\right),
$$

and eigenvalues

$$
\lambda_{n}=n\left(n-2 r_{1}-2 r_{2}+1\right) .
$$

We consider the parametrization

$$
r_{1}=\frac{N+1}{2}, \quad \alpha^{*}=\beta^{*}=-r_{1}-r_{2},
$$


where $N$ is an even or odd integer. It is seen from (3.8) and (3.10) that (6.3) is an admissible truncation condition for both parities of $N$. Upon introducing the variable $\widetilde{x}=x+r_{1}-1 / 2$, the coefficients of the eigenvalue equation (6.2) become

$$
B(x)=(\widetilde{x}-N)\left(\widetilde{x}+\alpha^{*}+1\right), \quad D(x)=\widetilde{x}\left(\widetilde{x}-\beta^{*}-N-1\right),
$$

and the eigenvalues have the expression

$$
\lambda_{n}=n\left(n+\alpha^{*}+\beta^{*}+1\right) .
$$

This corresponds to the difference equation of the Hahn polynomials [13]. With the parametrization (6.1) and (6.3), the recurrence relation (3.3) of the CBI polynomials becomes

$$
I_{n+1}(x)+\omega_{n} I_{n-1}(x)=x I_{n}(x),
$$

where

$$
\omega_{n}=\frac{n(N-n+1)\left(n+\alpha^{*}+\beta^{*}\right)\left(n+\alpha^{*}+\beta^{*}+N+1\right)}{4\left(2 n+\alpha^{*}+\beta^{*}-1\right)\left(2 n+\alpha^{*}+\beta^{*}+1\right)},
$$

which is indeed the recurrence relation of symmetric Hahn polynomials. A simple calculation shows that upon taking the parametrization (6.3) in structure parameters (5.6), one has

$$
\delta_{2}=\delta_{3}=\delta_{4}=0,
$$

and hence the algebra reduces to

$$
\begin{array}{ll}
{\left[K_{1}, P\right]=0, \quad\left\{K_{2}, P\right\}=0, \quad\left\{K_{3}, P\right\}=0, \quad\left[K_{1}, K_{2}\right]=K_{3},} \\
{\left[K_{1}, K_{3}\right]=\frac{1}{2}\left\{K_{1}, K_{2}\right\}+\delta_{1} K_{2}, \quad\left[K_{3}, K_{2}\right]=\frac{1}{2} K_{2}^{2}+K_{1}+\delta_{5},}
\end{array}
$$

with the remaining structure parameters

$$
\delta_{1}=\frac{1}{4}\left(r_{1}+r_{2}\right), \quad \delta_{5}=\frac{1}{4}\left(r_{1}-1 / 2\right)\left(r_{2}-1 / 2\right) .
$$

Thus we recover the Hahn algebra since the involution $P$ no longer plays a determining role. For reference, we record the two following alternate choices of CBI parameters which also lead to symmetric Hahn polynomials

$$
\rho_{2}=r_{1}=0, \quad \alpha=\frac{1}{4}\left(2 \rho_{1}-2 r_{2}+3\right), \quad \text { or } \quad \rho_{2}=r_{2}=0, \quad \alpha=\frac{1}{4}\left(2 \rho_{1}-2 r_{1}+3\right) .
$$

\subsection{Para-Krawtchouk polynomials}

The para-Krawtchouk polynomials have been found [28] in the design of spin chains effecting perfect quantum state transfer. These polynomials are directly connected to the complementary Bannai-Ito polynomials through the identification

$$
\rho_{1}=\frac{\gamma-N-3}{4}, \quad \rho_{2}=0, \quad r_{1}=\frac{N+1+\gamma}{4}, \quad r_{2}=0,
$$

when $N$ is a positive odd integer. (When $N$ is an even integer, the para-Krawtchouk are directly related to the Bannai-Ito polynomials.) In [28], the eigenvalue equation for the para-Krawtchouk polynomials was found; this operator corresponds to the operator (4.9) with a specific value of the free parameter

$$
\alpha=\frac{1-N}{4} .
$$

It is interesting to note that in the case $\rho_{2}=0$, the CBI polynomials and their descendants become symmetric and thus the "hidden" eigenvalue equation (4.5) appears trivial. Notwithstanding this, the corresponding symmetric polynomials are still eigenfunctions of a Dunkl operator with a free parameter in the odd sector of the spectrum. 


\section{Conclusion}

We have presented a systematic study of the complementary Bannai-Ito polynomials. We showed that these OPs are eigenfunctions of a one-parameter family of second order Dunkl shift operators and that in consequence they satisfy a one-parameter family of five-term difference equations on grids of the Bannai-Ito type. This result makes explicit the bispectrality of the CBI polynomials and places this OPs family outside the scope of the Leonard duality. Moreover, we have obtained the algebraic structure associated to the CBI polynomials which we named the complementary Bannai-Ito algebra. It was observed that this quadratic algebra is a deformation of the Askey-Wilson algebra with an involution. Lastly, we identified how the CBI polynomials are related to three other families of OPs.

The investigation of the continuum limit of the BI polynomials has led to connections with other families of -1 orthogonal polynomials which satisfy first order differential/Dunkl equations. It is hence of interest to examine the continuum limit of the CBI polynomials; this question will be treated in a future publication.

\section{Acknowledgements}

V.X.G. holds a scholarship from Fonds de recherche québécois - nature et technologies (FRQNT). The research of L.V. is supported in part by the Natural Science and Engineering Council of Canada (NSERC). A.Z. would like to thank the Centre de Recherches Mathématiques (CRM) for its hospitality.

\section{References}

[1] Bannai E., Ito T., Algebraic combinatorics. I. Association schemes, The Benjamin/Cummings Publishing Co. Inc., Menlo Park, CA, 1984.

[2] Chihara T.S., An introduction to orthogonal polynomials, Mathematics and its Applications, Vol. 13, Gordon and Breach Science Publishers, New York, 1978.

[3] Chihara T.S., On kernel polynomials and related systems, Boll. Un. Mat. Ital. (3) 19 (1964), 451-459.

[4] Dunkl C.F., Integral kernels with reflection group invariance, Canad. J. Math. 43 (1991), 1213-1227.

[5] Gasper G., Rahman M., Basic hypergeometric series, Encyclopedia of Mathematics and its Applications, Vol. 35, Cambridge University Press, Cambridge, 1990.

[6] Genest V.X., Vinet L., Zhedanov A., The algebra of dual -1 Hahn polynomials and the Clebsch-Gordan problem of $s l_{-1}(2)$, J. Math. Phys. 54 (2013), 023506, 13 pages, arXiv:1207.4220.

[7] Genest V.X., Vinet L., Zhedanov A., The Bannai-Ito polynomials as Racah coefficients of the $s l_{-1}(2)$ algebra, Proc. Amer. Math. Soc. to appear, arXiv:1205.4215.

[8] Granovskiı̌ Y.I., Lutzenko I.M., Zhedanov A.S., Mutual integrability, quadratic algebras, and dynamical symmetry, Ann. Physics 217 (1992), 1-20.

[9] Jafarov E.I., Stoilova N.I., Van der Jeugt J., Finite oscillator models: the Hahn oscillator, J. Phys. A: Math. Theor. 44 (2011), 265203, 15 pages, arXiv:1101.5310.

[10] Jafarov E.I., Stoilova N.I., Van der Jeugt J., The $\mathfrak{s u}(2)_{\alpha}$ Hahn oscillator and a discrete Fourier-Hahn transform, J. Phys. A: Math. Theor. 44 (2011), 355205, 18 pages, arXiv:1106.1083.

[11] Karlin S., McGregor J.L., The differential equations of birth-and-death processes, and the Stieltjes moment problem, Trans. Amer. Math. Soc. 85 (1957), 489-546.

[12] Karlin S., McGregor J.L., The Hahn polynomials, formulas and an application, Scripta Math. 26 (1961), $33-46$.

[13] Koekoek R., Lesky P.A., Swarttouw R.F., Hypergeometric orthogonal polynomials and their $q$-analogues, Springer Monographs in Mathematics, Springer-Verlag, Berlin, 2010.

[14] Leonard D.A., Orthogonal polynomials, duality and association schemes, SIAM J. Math. Anal. 13 (1982), 656-663. 
[15] Marcellán F., Petronilho J., Eigenproblems for tridiagonal 2-Toeplitz matrices and quadratic polynomial mappings, Linear Algebra Appl. 260 (1997), 169-208.

[16] Szegö G., Orthogonal polynomials, American Mathematical Society, Colloquium Publications, Vol. 23, 4th ed., Amer. Math. Soc., Providence, R.I., 1975.

[17] Terwilliger P., The universal Askey-Wilson algebra and the equitable presentation of $U_{q}\left(s l_{2}\right), S I G M A \mathbf{7}$ (2011), 099, 26 pages, arXiv:1107.3544.

[18] Terwilliger P., Two linear transformations each tridiagonal with respect to an eigenbasis of the other, Linear Algebra Appl. 330 (2001), 149-203, math.RA/0406555.

[19] Terwilliger P., Vidunas R., Leonard pairs and the Askey-Wilson relations, J. Algebra Appl. 3 (2004), 411426, math.QA/0305356.

[20] Tsujimoto S., Vinet L., Zhedanov A., Dual -1 Hahn polynomials: "classical" polynomials beyond the Leonard duality, Proc. Amer. Math. Soc. 141 (2013), 959-970, arXiv:1108.0132.

[21] Tsujimoto S., Vinet L., Zhedanov A., Dunkl shift operators and Bannai-Ito polynomials, Adv. Math. 229 (2012), 2123-2158, arXiv:1106.3512.

[22] Tsujimoto S., Vinet L., Zhedanov A., From $s l_{q}(2)$ to a parabosonic Hopf algebra, SIGMA 7 (2011), 093, 13 pages, arXiv:1108.1603.

[23] Vidunas R., Askey-Wilson relations and Leonard pairs, Discrete Math. 308 (2008), 479-495, math.QA/0511509.

[24] Vinet L., Zhedanov A., A Bochner theorem for Dunkl polynomials, SIGMA 7 (2011), 020, 9 pages, arXiv:1011.1457.

[25] Vinet L., Zhedanov A., A limit $q=-1$ for the big $q$-Jacobi polynomials, Trans. Amer. Math. Soc. 364 (2012), 5491-5507, arXiv:1011.1429.

[26] Vinet L., Zhedanov A., A 'missing' family of classical orthogonal polynomials, J. Phys. A: Math. Theor. 44 (2011), 085201, 16 pages, arXiv:1011.1669.

[27] Vinet L., Zhedanov A., Dual -1 Hahn polynomials and perfect state transfer, J. Phys. Conf. Ser. 343 (2012), 012125, 10 pages, arXiv:1110.6477.

[28] Vinet L., Zhedanov A., Para-Krawtchouk polynomials on a bi-lattice and a quantum spin chain with perfect state transfer, J. Phys. A: Math. Theor. 45 (2012), 265304, 11 pages, arXiv:1110.6475.

[29] Zhedanov A., "Hidden symmetry" of Askey-Wilson polynomials, Theoret. and Math. Phys. 89 (1991), 1146-1157.

[30] Zhedanov A., Rational spectral transformations and orthogonal polynomials, J. Comput. Appl. Math. 85 (1997), 67-86.

[31] Zhedanov A., The "Higgs algebra" as a "quantum" deformation of SU(2), Modern Phys. Lett. A 7 (1992), $507-512$. 